Research Article; Received: January 15, 2021; Accepted: July 29, 2021

\title{
ON A NONLINEAR FUZZY DIFFERENCE EQUATION
}

\author{
İbrahim YALÇINKAYA ${ }^{1}$, Vildan ÇALIŞKAN ${ }^{2}$, and Durhasan Turgut TOLLU ${ }^{1}$ \\ ${ }^{1}$ Department of Mathematics and Computer Sciences, Necmettin Erbakan University, \\ Konya, TURKEY \\ ${ }^{2}$ Graduate School of Natural and Applied Science, Necmettin Erbakan University, \\ Konya, TURKEY
}

\begin{abstract}
In this paper we investigate the existence, the boundedness and the asymptotic behavior of the positive solutions of the fuzzy difference equation$$
z_{n+1}=\frac{A z_{n-1}}{1+z_{n-2}^{p}}, n \in \mathbb{N}_{0}
$$

where $\left(z_{n}\right)$ is a sequence of positive fuzzy numbers, $A$ and the initial conditions $z_{-j}(j=0,1,2)$ are positive fuzzy numbers and $p$ is a positive integer.
\end{abstract}

\section{INTRODUCTION}

Over the last two decades, a lot of study has been published on difference equations and systems. One reason for this is that such equations and systems have high applicability both in mathematics and other sciences such as population biology, economics, probability theory, genetics, psychology etc., (see, e.g., 2, 6, 14,15 and the references therein). In this way, many real life problems are modeled by means of difference equations and systems. In some cases, however, measurements or data on a problem may reveal uncertainty or the problem considered may require subjective interpretations. In such cases, a fuzzy difference equation model can be established using notion of fuzzy set. In this way, the uncertainty is modeled.

Fuzzy set theory has recently become a popular subject due to the increasing number of applications in technology, mathematics and other sciences. The part that we are interested in is, of course, that the notion of fuzzy set can be easily

2020 Mathematics Subject Classification. 39A10, 39A26, 39A30.

Keywords. Asymptotic behavior, $\alpha$-cuts, boundedness, fuzzy difference equations, fuzzy number, stability.

■iyalcinkaya@erbakan.edu.tr-Corresponding author; clskn.vldn95@gmail.com; dttollu@erbakan.edu.tr

(D) 0000-0003-4546-4493; 0000-0003-4763-1689; 0000-0002-3313-8829. 
applied to difference equations. With this application, a powerful method for determining the behavior of solutions of difference equations emerges. Some studies using the method will be summarized below.

In [3], Deeba et al. studied the fuzzy analog of a difference equation which arises in population genetics. More precisely they studied the first order difference equation

$$
x_{n+1}=w x_{n}+q, n \in \mathbb{N}_{0}
$$

where $\left(x_{n}\right)$ is a sequence of fuzzy numbers and $w, q, x_{0}$ are fuzzy numbers. Also, they discussed the fuzzy nonlinear difference equation

$$
x_{n+1}=f\left(x_{n}, w, q\right), n \in \mathbb{N}_{0}
$$

where $\left(x_{n}\right)$ is a sequence of fuzzy numbers, $w, q, x_{0}$ are fuzzy numbers and $f$ : $\mathbb{R}_{a}^{+} \times \mathbb{R}_{a}^{+} \times \mathbb{R}_{a}^{+} \rightarrow \mathbb{R}_{a}^{+}, \mathbb{R}_{a}^{+}$is the set of all real numbers greater or equal to $a$, is a continuous and nondecreasing function in its arguments.

In [4], Deeba and Korvin studied the second order difference equation

$$
x_{n+1}=x_{n}-a b x_{n-1}+c, n \in \mathbb{N}_{0}
$$

where $\left(x_{n}\right)$ is a sequence of fuzzy numbers and $a, b, c, x_{0}, x_{-1}$ are fuzzy numbers. This equation is a linearized model of a nonlinear model which determines the carbondioxide $\left(\mathrm{CO}_{2}\right)$ level in the blood.

In 12], Papaschinopoulos and Papadopoulos studied the existence, the boundedness and the asymptotic behavior of the positive solutions of the fuzzy difference equation

$$
x_{n+1}=A+\frac{x_{n}}{x_{n-m}}, n \in \mathbb{N}_{0}
$$

where $\left(x_{n}\right)$ is a sequence of fuzzy numbers and $A$ and the initial conditions $x_{-j}$ $(j=0,1, \ldots, m)$ are fuzzy numbers for $m \in \mathbb{N}_{1}$.

For more works on fuzzy difference equations, see the references [9, 11, 13 and the references cited therein.

In [5], El-Owaidy et al. investigated the global behavior of the difference equation

$$
x_{n+1}=\frac{\alpha x_{n-1}}{\beta+\gamma x_{n-2}^{p}}, n \in \mathbb{N}_{0}
$$

where the nonnegative parameters and nonnegative initial conditions.

Moreover, in [7], Gümüş and Soykan investigated the behavior of solutions of the system of difference equations

$$
u_{n+1}=\frac{\alpha u_{n-1}}{\beta+\gamma v_{n-2}^{p}}, v_{n+1}=\frac{\alpha_{1} v_{n-1}}{\beta_{1}+\gamma_{1} u_{n-2}^{p}}, n \in \mathbb{N}_{0}
$$

where the positive parameters $\alpha, \beta, \gamma, \alpha_{1}, \beta_{1}, \gamma_{1}, p$ and the initial conditions $u_{-i}, v_{-i}$ for $i=0,1,2$ are positive real numbers. Note that system (6) can be reduced to the following system of difference equations 


$$
x_{n+1}=\frac{r x_{n-1}}{1+y_{n-2}^{p}}, y_{n+1}=\frac{s y_{n-1}}{1+x_{n-2}^{p}}, n \in \mathbb{N}_{0}
$$

by the change of variables $u_{n}=\left(\beta_{1} / \gamma_{1}\right)^{1 / p} x_{n}$ and $v_{n}=(\beta / \gamma)^{1 / p} y_{n}$ with $r=\alpha / \beta$ and $s=\alpha_{1} / \beta_{1}$. So, in order to study system (6), they investigated system (7).

In this paper we investigate the existence, the boundedness and the asymptotic behavior of the positive solutions of the fuzzy difference equation

$$
z_{n+1}=\frac{A z_{n-1}}{1+z_{n-2}^{p}}, n \in \mathbb{N}_{0}
$$

where $\left(z_{n}\right)$ is a sequence of positive fuzzy numbers, $A$ and the initial conditions $z_{-j}$ $(j=0,1,2)$ are positive fuzzy numbers and $p$ is a positive integer.

\section{Preliminaries}

In this section, we give some definitions which will be used in this paper. For more details see $1,8,10,16$.

Definition 1. Consider a fuzzy subset of the real line $A: \mathbb{R} \rightarrow[0,1]$. Then we say $A$ is a fuzzy number if it is satisfies the following properties

(a) $A$ is normal, i.e., $\exists x_{0} \in \mathbb{R}$ with $A\left(x_{0}\right)=1$,

(b) $A$ is fuzzy convex, i.e., $A\left(t x_{1}+(1-t) x_{2}\right) \geq \min \left\{A\left(x_{1}\right), A\left(x_{2}\right)\right\}, \forall t \in[0,1]$ and $x_{1}, x_{2} \in \mathbb{R}$

(c) $A$ is upper semicontinuous on $\mathbb{R}$,

(d) $A$ is compactly supported, i.e., $\overline{\{x \in \mathbb{R}: A(x)>0\}}$ is compact.

Let us denote by $\mathbb{R}_{F}$ the space of all fuzzy numbers. For $0<\alpha \leq 1$ and $A \in \mathbb{R}_{F}$, we denote $\alpha$-cuts of fuzzy number $A$ by $[A]_{\alpha}=\{x \in \mathbb{R}, A(x) \geq \alpha\}$ and $[A]_{0}=\overline{\{x \in \mathbb{R}, A(x) \geq 0\}}$. We call $[A]_{0}$, the support of fuzzy number $A$ and denote it by $\operatorname{supp}(A)$.

The fuzzy number $A$ is called positive if $\operatorname{supp}(A) \subset(0, \infty)$. We denote by $\mathbb{R}_{F}^{+}$, the space of all positive fuzzy numbers.

Definition 2. (a) Let $A, B$ be any fuzzy numbers with $[A]_{\alpha}=\left[A_{l, \alpha}, A_{r, \alpha}\right]$ and $[B]_{\alpha}=\left[B_{l, \alpha}, B_{r, \alpha}\right]$ for $\alpha \in(0,1]$. We define $\|A\|$ on the fuzzy numbers space as follow;

$$
\|A\|=\sup \max \left\{\left|A_{l, \alpha}\right|,\left|A_{r, \alpha}\right|\right\},
$$

where sup is taken for all $\alpha \in(0,1]$. Then from the above we take the following metric

$$
D(A, B)=\sup \left\{\max \left\{\left|A_{l, \alpha}-B_{l, \alpha}\right|,\left|A_{r, \alpha}-B_{r, \alpha}\right|\right\}\right\},
$$

where sup is taken for all $\alpha \in(0,1]$.

(b) Let $\left(x_{n}\right)$ be a sequence of positive fuzzy numbers and $x$ is a fuzzy number. Then we say that

$$
\lim _{n \rightarrow \infty} x_{n}=x \quad \text { iff } \quad \lim _{n \rightarrow \infty} D\left(x_{n}, x\right)=0 .
$$


The following lemma and definition are given in 8 :

Lemma 1. Let $X, Y$ be fuzzy numbers and $[X]_{\alpha}=\left[X_{l, \alpha}, X_{r, \alpha}\right],[Y]_{\alpha}=\left[Y_{l, \alpha}, Y_{r, \alpha}\right]$ for $\alpha \in(0,1]$ be the $\alpha$-cuts of $X, Y$, respectively. Let $Z$ be a fuzzy number such that $[Z]_{\alpha}=\left[Z_{l, \alpha}, Z_{r, \alpha}\right]$ for $\alpha \in(0,1]$. Then, $M I N\{X, Y\}=Z$ (resp. $M A X\{X, Y\}=$ $Z$ ) if and only if $\min \left\{X_{l, \alpha}, Y_{l, \alpha}\right\}=Z_{l, \alpha}$ and $\min \left\{X_{r, \alpha}, Y_{r, \alpha}\right\}=Z_{r, \alpha}$ (resp. $\max \left\{X_{l, \alpha}, Y_{l, \alpha}\right\}=Z_{l, \alpha}$ and $\left.\max \left\{X_{r, \alpha}, Y_{r, \alpha}\right\}=Z_{r, \alpha}\right)$.

Definition 3. (a) We say that a sequence of positive fuzzy numbers $\left(x_{n}\right)$ is bounded and persistent if there exist $n_{0} \in \mathbb{N}$ and positive fuzzy numbers $C, D$ such that $M I N\left\{x_{n}, C\right\}=C$ and $M I N\left\{x_{n}, D\right\}=D$ for $n \geq n_{0}$.

(b) We say that $\left(x_{n}\right)$ for $n \in \mathbb{N}_{0}$ is an unbounded sequence if the $\left\|x_{n}\right\|$ for $n \in \mathbb{N}_{0}$ is an unbounded sequence.

We need the following lemma which has been proved in 12 .

Lemma 2. Let $f: \mathbb{R}^{+} \times \mathbb{R}^{+} \times \mathbb{R}^{+} \rightarrow \mathbb{R}^{+}$be a continuous function and $A, B, C$ be fuzzy numbers. Then, $[f(A, B, C)]_{\alpha}=f\left([A]_{\alpha},[B]_{\alpha},[C]_{\alpha}\right)$ for $\alpha \in(0,1]$.

\section{Main Results}

In this section, we prove our main results. Firstly, we will study the existence of the positive solutions of equation (8). We say $\left(z_{n}\right)$ is a positive solution of equation (8) if $\left(z_{n}\right)$ is a sequence of positive fuzzy numbers which satisfies equation (8).

Theorem 1. Consider equation (8) where $A$ is a positive fuzzy number. Then for any positive fuzzy numbers $z_{-j}(j=0,1,2)$ there exists a unique positive solution $\left(z_{n}\right)$ of (8) with the initial conditions $z_{-j}(j=0,1,2)$.

Proof. Suppose that there exists a sequence of fuzzy numbers $\left(z_{n}\right)$ satisfying 8 with the initial conditions $z_{-j}(j=0,1,2)$. Consider the $\alpha$-cuts

$$
\begin{gathered}
{\left[z_{n}\right]_{\alpha}=\left[L_{n, \alpha}, R_{n, \alpha}\right],} \\
{[A]_{\alpha}=\left[A_{l, \alpha}, A_{r, \alpha}\right],}
\end{gathered}
$$

for $n=-2,-1,0, \ldots$ and $\alpha \in(0,1]$. Then from $(8)-(9)$ and Lemma 2 it follows that

$$
\begin{aligned}
{\left[z_{n+1}\right]_{\alpha} } & =\left[L_{n+1, \alpha}, R_{n+1, \alpha}\right] \\
& =\left[\frac{A z_{n-1}}{1+z_{n-2}^{p}}\right]_{\alpha}=\frac{[A]_{\alpha}\left[z_{n-1}\right]_{\alpha}}{\left[1+z_{n-2}^{p}\right]_{\alpha}} \\
& =\frac{\left[A_{l, \alpha} L_{n-1, \alpha}, A_{r, \alpha} R_{n-1, \alpha}\right]}{\left[1+L_{n-2, \alpha}^{p}, 1+R_{n-2, \alpha}^{p}\right]}=\left[\frac{A_{l, \alpha} L_{n-1, \alpha}}{1+R_{n-2, \alpha}^{p}}, \frac{A_{r, \alpha} R_{n-1, \alpha}}{1+L_{n-2, \alpha}^{p}}\right],
\end{aligned}
$$

from which we get

$$
L_{n+1, \alpha}=\frac{A_{l, \alpha} L_{n-1, \alpha}}{1+R_{n-2, \alpha}^{p}}, R_{n+1, \alpha}=\frac{A_{r, \alpha} R_{n-1, \alpha}}{1+L_{n-2, \alpha}^{p}}, \alpha \in(0,1] .
$$


for $n \in \mathbb{N}_{0}$. Then it is clear that for any $\left(L_{j, \alpha}, R_{j, \alpha}\right), j=-2,-1,0$ there exists a unique solution $\left(L_{n, \alpha}, R_{n, \alpha}\right)$ with the initial conditions $\left(L_{j, \alpha}, R_{j, \alpha}\right), j=-2,-1,0$ for $\alpha \in(0,1]$.

Now, we prove that $\left[L_{n, \alpha}, R_{n, \alpha}\right]$ for $\alpha \in(0,1]$ where $\left(L_{n, \alpha}, R_{n, \alpha}\right)$ is the solution of the system 10 with the initial conditions $\left(L_{j, \alpha}, R_{j, \alpha}\right), j=-2,-1,0$ determines the solution $\left(z_{n}\right)$ of $(8)$ with the initial conditions $z_{-j}(j=0,1,2)$ such that

$$
\left[z_{n}\right]_{\alpha}=\left[L_{n, \alpha}, R_{n, \alpha}\right], \alpha \in(0,1], n=-2,-1,0, \ldots
$$

Since $A$ and $z_{-j}(j=0,1,2)$ are positive fuzzy numbers for any $\alpha_{1}, \alpha_{2} \in(0,1]$, $\alpha_{1} \leq \alpha_{2}$ we get,

$$
\begin{aligned}
0<A_{l, \alpha_{1}} \leq A_{l, \alpha_{2}} & \leq A_{r, \alpha_{2}} \leq A_{r, \alpha_{1}} \\
0<L_{-2, \alpha_{1}} \leq L_{-2, \alpha_{2}} & \leq R_{-2, \alpha_{2}} \leq R_{-2, \alpha_{1}}, \\
0<L_{-1, \alpha_{1}} \leq L_{-1, \alpha_{2}} & \leq R_{-1, \alpha_{2}} \leq R_{-1, \alpha_{1}} \\
0<L_{0, \alpha_{1}} \leq L_{0, \alpha_{2}} & \leq R_{0, \alpha_{2}} \leq R_{0, \alpha_{1}}
\end{aligned}
$$

We prove by the induction that

$$
L_{n, \alpha_{1}} \leq L_{n, \alpha_{2}} \leq R_{n, \alpha_{2}} \leq R_{n, \alpha_{1}}, n \in \mathbb{N} .
$$

From (12) we have that (13) hold for $n=-2,-1,0$. Suppose that (13) is valid for $n \leq k, k \in\{1,2, \ldots\}$. Then from $\sqrt{10}, \sqrt{12}$ and $(13)$ for $n \leq k$ it follows that

$$
\begin{aligned}
& L_{k+1, \alpha_{1}}=\frac{A_{l, \alpha_{1}} L_{k-1, \alpha_{1}}}{1+R_{k-2, \alpha_{1}}^{p}} \leq \frac{A_{l, \alpha_{2}} L_{k-1, \alpha_{2}}}{1+R_{k-2, \alpha_{2}}^{p}}=L_{k+1, \alpha_{2}}, \\
& L_{k+1, \alpha_{2}}=\frac{A_{l, \alpha_{2}} L_{k-1, \alpha_{2}}}{1+R_{k-2, \alpha_{2}}^{p}} \leq \frac{A_{r, \alpha_{2}} R_{k-1, \alpha_{2}}}{1+L_{k-2, \alpha_{2}}^{p}}=R_{k+1, \alpha_{2}}
\end{aligned}
$$

and

$$
R_{k+1, \alpha_{2}}=\frac{A_{r, \alpha_{2}} R_{k-1, \alpha_{2}}}{1+L_{k-2, \alpha_{2}}^{p}} \leq \frac{A_{r, \alpha_{1}} R_{k-1, \alpha_{1}}}{1+L_{k-2, \alpha_{1}}^{p}}=R_{k+1, \alpha_{1}} .
$$

Therefore 13 is satisfied. Moreover from 10 we get

$$
L_{1, \alpha}=\frac{A_{l, \alpha} L_{-1, \alpha}}{1+R_{-2, \alpha}^{p}}, R_{1, \alpha}=\frac{A_{r, \alpha} R_{-1, \alpha}}{1+L_{-2, \alpha}^{p}}, \alpha \in(0,1] .
$$

Then since $A$ and $z_{-j}(j=0,1,2)$ are positive fuzzy numbers, we have that $A_{l, \alpha}$, $A_{r, \alpha}, L_{-1, \alpha}, R_{-1, \alpha}, L_{-2, \alpha}$ and $R_{-2, \alpha}$ are left continuous. So, from (14) we see that $L_{1, \alpha}$ and $R_{1, \alpha}$ are also left continuous. Working inductively we can easily prove that $L_{n, \alpha}$ and $R_{n, \alpha}$ are left continuous for $n \in \mathbb{N}$.

Now, we prove that $\overline{\cup_{\alpha \in(0,1]}\left[L_{n, \alpha}, R_{n, \alpha}\right]}$ is compact. It is sufficient to prove that $\cup_{\alpha \in(0,1]}\left[L_{n, \alpha}, R_{n, \alpha}\right]$ is bounded. Let $n=1$, since $A$ and $z_{-j}(j=0,1,2)$ are positive fuzzy numbers there exist constants $M_{A}, N_{A}, M_{-2}, N_{-2}, M_{-1}, N_{-1}, M_{0}, N_{0}>0$ 
such that

$$
\begin{aligned}
{\left[A_{l, \alpha}, A_{r, \alpha}\right] } & \subset\left[M_{A}, N_{A}\right], \\
{\left[L_{-2, \alpha}, R_{-2, \alpha}\right] } & \subset\left[M_{-2}, N_{-2}\right], \\
{\left[L_{-1, \alpha}, R_{-1, \alpha}\right] } & \subset\left[M_{-1}, N_{-1}\right], \\
{\left[L_{0, \alpha}, R_{0, \alpha}\right] } & \subset\left[M_{0}, N_{0}\right] .
\end{aligned}
$$

Therefore, from (14)-(15) we can easily prove that

$$
\left[L_{1, \alpha}, R_{1, \alpha}\right] \subset\left[\frac{M_{A} M_{-1}}{1+N_{-2}^{p}}, \frac{N_{A} N_{-1}}{1+M_{-2}^{p}}\right],
$$

for $\alpha \in(0,1]$ from which it is clear that

$$
\cup_{\alpha \in(0,1]}\left[L_{1, \alpha}, R_{1, \alpha}\right] \subset\left[\frac{M_{A} M_{-1}}{1+N_{-2}^{p}}, \frac{N_{A} N_{-1}}{1+M_{-2}^{p}}\right],
$$

for $\alpha \in(0,1]$. Also, $\sqrt{16})$ implies that $\overline{\cup_{\alpha \in(0,1]}\left[L_{1, \alpha}, R_{1, \alpha}\right]}$ is compact and

$$
\overline{\cup_{\alpha \in(0,1]}\left[L_{1, \alpha}, R_{1, \alpha}\right]} \subset(0, \infty) .
$$

Working inductively we can easily see that

$$
\overline{\cup_{\alpha \in(0,1]}\left[L_{n, \alpha}, R_{n, \alpha}\right]} \text { is compact, } \overline{\cup_{\alpha \in(0,1]}\left[L_{n, \alpha}, R_{n, \alpha}\right]} \subset(0, \infty) \text { for } n \in \mathbb{N}_{1} \text {. }
$$

Therefore, using (13), 17) and since $L_{n, \alpha}, R_{n, \alpha}$ are left continuous we see that $\left[L_{n, \alpha}, R_{n, \alpha}\right]$ determines a sequence of positive fuzzy numbers $\left(z_{n}\right)$ such that (11) holds.

We prove now that $\left(z_{n}\right)$ is the solution of equation (8) with the initial conditions $z_{-j}(j=0,1,2)$. Since

$$
\left[z_{n+1}\right]_{\alpha}=\left[L_{n+1, \alpha}, R_{n+1, \alpha}\right]=\left[\frac{A_{l, \alpha} L_{n-1, \alpha}}{1+R_{n-2, \alpha}^{p}}, \frac{A_{r, \alpha} R_{n-1, \alpha}}{1+L_{n-2, \alpha}^{p}}\right]=\left[\frac{A z_{n-1}}{1+z_{n-2}^{p}}\right]_{\alpha},
$$

for all $\alpha \in(0,1]$, we have that $\left(z_{n}\right)$ is the solution of equation $(8)$ with the initial conditions $z_{-j}(j=0,1,2)$.

Suppose that there exists another solution $\left(\widetilde{z}_{n}\right)$ of equation (8) with the initial conditions $z_{-j}(j=0,1,2)$. Then arguing as above we can easily show that

$$
\left[\widetilde{z}_{n}\right]_{\alpha}=\left[L_{n, \alpha}, R_{n, \alpha}\right] \text { for } \alpha \in(0,1] \text { and } n \in \mathbb{N}_{0} .
$$

Then from (11) and (18) we have that $\left[z_{n}\right]_{\alpha}=\left[\widetilde{z}_{n}\right]_{\alpha}$ for $\alpha \in(0,1]$ and $n=$ $-2,-1,0, \ldots$ from which it holds $z_{n}=\widetilde{z}_{n}$ for $n=-2,-1,0, \ldots$ Thus, the proof is completed.

To study the boundedness of the positive solutions of equation (8), we need the following theorem which has been proved in 7 :

Theorem 2. Assume that $r, s \in(1, \infty)$, then there exist unbounded solutions of system (7).

In the following lemma, we will study the boundedness and persistence of the positive solutions of system (7): 
Lemma 3. Assume that $r, s \in(0,1)$, then every positive solution of system (7) is bounded and persists.

Proof. Assume that $r, s \in(0,1)$. From system (7), we have that

$$
0<x_{n+1}=\frac{r x_{n-1}}{1+y_{n-2}^{p}}<r x_{n-1}<x_{n-1}
$$

and

$$
0<y_{n+1}=\frac{s y_{n-1}}{1+x_{n-2}^{p}}<s y_{n-1}<y_{n-1},
$$

for $n \in \mathbb{N}_{0}$. From $(19)$ and $(20)$, we have by induction

$$
0<x_{2 n-i}<x_{-i} \text { and } 0<y_{2 n-i}<y_{-i},
$$

for $n \in \mathbb{N}_{1}$ where $x_{-i}, y_{-i}(i=0,1)$ are the initial conditions. This completes the proof.

Theorem 3. Consider equation (8). Then the following statements are true:

(i) If $A_{r, \alpha}<1$ for all $\alpha \in(0,1]$, then every positive solution of equation (8) is bounded and persists.

(ii) If there exists an $\bar{\alpha} \in(0,1]$ such that $A_{l, \bar{\alpha}}>1$, then the equation (8) has unbounded solutions.

Proof. (i) Let $\left(z_{n}\right)$ be a positive solution of equation (8) such that (11) holds. From 10) and Lemma 3 , we get

$$
\left[L_{n, \alpha}, R_{n, \alpha}\right] \subset\left[0, T_{\alpha}\right],
$$

for $n \in \mathbb{N}_{1}$ where $T_{\alpha}=\max \left\{R_{-1, \alpha}, R_{0, \alpha}\right\}$. Since $\left(z_{n}\right)$ are positive fuzzy numbers, there exists a constant $T>0$ such that $T_{\alpha} \leq T$ for all $\alpha \in(0,1]$. Therefore, $\left[L_{n, \alpha}, R_{n, \alpha}\right] \subset[0, T]$ for $n \in \mathbb{N}_{1}$ from which we get $\cup_{\alpha \in(0,1]}\left[L_{n, \alpha}, R_{n, \alpha}\right] \subset[0, T]$ for $n \in \mathbb{N}_{1}$ so $\overline{\cup_{\alpha \in(0,1]}\left[L_{n, \alpha}, R_{n, \alpha}\right]} \subseteq[0, T]$. This completes the proof of $(i)$.

(ii) Suppose that there exists an $\bar{\alpha} \in(0,1]$ such that $A_{l, \bar{\alpha}}>1$. If $A_{l, \bar{\alpha}}=r$, $A_{r, \bar{\alpha}}=s, L_{n, \bar{\alpha}}=x_{n}$ and $R_{n, \bar{\alpha}}=y_{n}$ for $n=-2,-1, \ldots$, then we can apply Theorem 2 to system $(10)$. If there exists an $\bar{\alpha} \in(0,1]$ such that $r=A_{l, \bar{\alpha}}>1$, then there exist solutions $\left(x_{n}, y_{n}\right)$ of system 10$)$ where $\bar{\alpha}=\alpha$ with initial conditions $\left(x_{-j}, y_{-j}\right)$ for $j=0,1,2$ such that

$$
\lim _{n \rightarrow \infty} x_{n}=0 \text { and } \lim _{n \rightarrow \infty} y_{n}=\infty .
$$

Moreover, if $x_{-j}<y_{-j}(j=0,1,2)$, we can find positive fuzzy numbers $z_{-j}$ $(j=0,1,2)$ such that

$$
\left[z_{j}\right]_{\alpha}=\left[L_{j, \alpha}, R_{j, \alpha}\right]
$$

for $\alpha \in(0,1]$ and

$$
\left[z_{j}\right]_{\bar{\alpha}}=\left[L_{j, \bar{\alpha}}, R_{j, \bar{\alpha}}\right]=\left[x_{j}, y_{j}\right],
$$


for $j=-2,-1,0$. Let $\left(z_{n}\right)$ be a positive solution of equation (8) with the initial conditions $z_{-j}(j=0,1,2)$ and $\left[z_{n}\right]_{\alpha}=\left[L_{n, \alpha}, R_{n, \alpha}\right]$ for $\alpha \in(0,1]$. Since $(24)$ and 25) hold and $\left(L_{n, \alpha}, R_{n, \alpha}\right)$ satisfies system 10) we have

$$
\left[z_{n}\right]_{\bar{\alpha}}=\left[L_{n, \bar{\alpha}}, R_{n, \bar{\alpha}}\right]=\left[x_{n}, y_{n}\right] .
$$

Thefore, from (23), 26) and since

$$
\| z_{n}||=\sup \max \left\{\left|L_{n, \alpha}\right|,\left|R_{n, \alpha}\right|\right\} \geq \max \left\{\left|L_{n, \bar{\alpha}}\right|,\left|R_{n, \bar{\alpha}}\right|\right\}=R_{n, \bar{\alpha}},
$$

where sup is taken for all $\alpha \in(0,1]$, it is clear that solution $\left(z_{n}\right)$ is unbounded. This completes the proof of $(i i)$.

In the last theorem, we will study the convergence of the positive solutions of equation (8). We need the following theorem which has been obtained from Theorem 10 in 7 :

Theorem 4. If $r, s \in(0,1)$, then every positive solution $\left(x_{n}, y_{n}\right)$ of system (7) converges to $(0,0)$ as $n \rightarrow \infty$.

Theorem 5. If $A_{r, \alpha}<1$ for all $\alpha \in(0,1]$, then every positive solution $\left(z_{n}\right)$ of equation (8) converges to 0 as $n \rightarrow \infty$.

Proof. Let $\left(z_{n}\right)$ be a positive solution of equation (8) such that $(9)$ holds with $A_{r, \alpha}<1$ for all $\alpha \in(0,1]$. Then, we can apply Theorem 4 to system 10$]$. So, we get

$$
\lim _{n \rightarrow \infty} L_{n, \alpha}=\lim _{n \rightarrow \infty} R_{n, \alpha}=0 .
$$

Therefore, from 27] we get

$$
\lim _{n \rightarrow \infty} D\left(z_{n}, 0\right)=\lim _{n \rightarrow \infty} \sup \left\{\max \left\{\left|L_{n, \alpha}-0\right|,\left|R_{n, \alpha}-0\right|\right\}\right\}=0 .
$$

\section{Numerical Examples}

In this section, we give two numerical examples for the solutions of equation (8) regard to the different values of $A$ for $p=1$ with the inital conditions $z_{-j}$ $(j=0,1,2)$ are satisfied

$$
\begin{aligned}
& z_{-2}(x)=\left\{\begin{array}{cc}
20 x-8, & 0.40 \leq x \leq 0.45 \\
10-20 x, & 0.45 \leq x \leq 0.50
\end{array}\right. \\
& z_{-1}(x)= \begin{cases}\frac{5 x-0.50}{2}, & 0.10 \leq x \leq 0.50 \\
\frac{4.50-5 x}{2}, & 0.50 \leq x \leq 0.90\end{cases} \\
& z_{0}(x)= \begin{cases}20 x-6, & 0.30 \leq x \leq 0.35 \\
8-20 x, & 0.35 \leq x \leq 0.40\end{cases}
\end{aligned}
$$


From $\left[28\right.$, we get $\left[z_{-2}\right]_{\alpha}=\left[\frac{\alpha+8}{20}, \frac{10-\alpha}{20}\right],\left[z_{-1}\right]_{\alpha}=\left[\frac{2 \alpha+0.50}{5}, \frac{4.50-2 \alpha}{5}\right],\left[z_{0}\right]_{\alpha}=$ $\left[\frac{\alpha+6}{20}, \frac{8-\alpha}{20}\right]$ for all $\alpha \in(0,1]$.

Example 1. Consider equation (8) for $p=1$ where $z_{n}$ is a sequence of positive fuzzy numbers, the initial conditions $z_{-j}(j=0,1,2)$ are satisfied (28) and $A$ is satisfied

$$
A= \begin{cases}4 x-1, & \frac{1}{4} \leq x \leq \frac{1}{2}, \\ 3-4 x, & \frac{1}{2} \leq x \leq \frac{3}{4} .\end{cases}
$$

From (29), we get $[A]_{\alpha}=\left[\frac{\alpha+1}{4}, \frac{3-\alpha}{4}\right]$ for all $\alpha \in(0,1]$. There exists a unique solution of equation (8) by Theorem 1 . Since $A_{r, \alpha}<1$ for all $\alpha \in[0,1]$, then by Theorem 5, the positive solution $\left(z_{n}\right)$ of equation (8) converges to 0 as $n \rightarrow \infty$, see Figures 1-3.

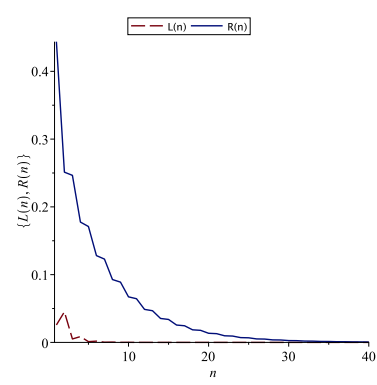

Figure

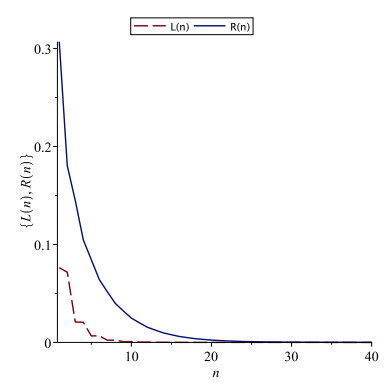

Figure

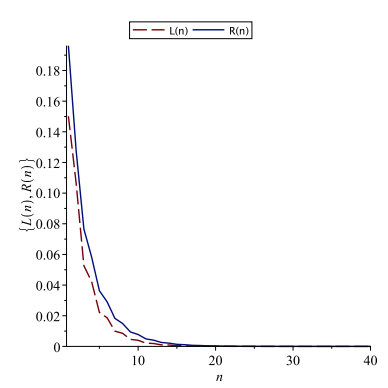

FIGURE

3. $\alpha=0.9$

Example 2. Consider equation (8) for $p=1$ where $z_{n}$ is a sequence of positive fuzzy numbers, the initial conditions $z_{-j}(j=0,1,2)$ are satisfied (28) and $A$ is satisfied

$$
A= \begin{cases}x-2, & 2 \leq x \leq 3 \\ 4-x, & 3 \leq x \leq 4\end{cases}
$$

From (30), we get $[A]_{\alpha}=[\alpha+2,4-\alpha]$ for all $\alpha \in(0,1]$. There exists a unique positive solution of equation (8) by Theorem 1 . It is easy to see that for all $\alpha \in(0,1]$, we have $A_{l, \alpha}>1$. So, by case (ii) in Theorem 3. equation (8) has unbounded solutions, see Figures 4-6. 


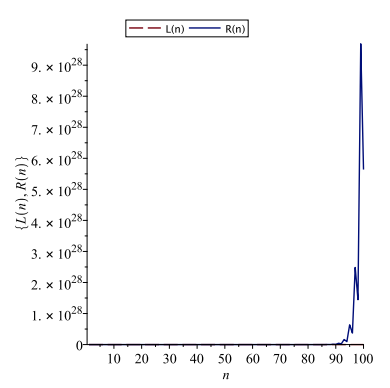

FIGURE

4. $\alpha=0.1$

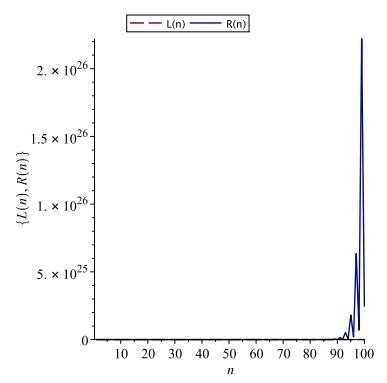

FIGURE

5. $\alpha=0.5$

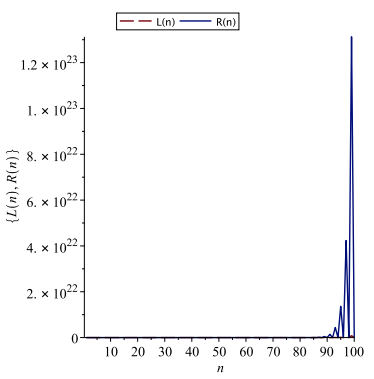

FIGURE

6. $\alpha=0.9$

\section{Conclusion}

In this study, we investigated behavior of the fuzzy difference equation $z_{n+1}=$ $A z_{n-1} /\left(1+z_{n-2}^{p}\right)$, where $\left(z_{n}\right)$ is a sequence of positive fuzzy numbers, $A$ and the initial conditions $z_{-j}(j=0,1,2)$ are positive fuzzy numbers and $p$ is a positive integer. We have shown that, under certain conditions, the positive solutions of this equation converge to zero. Also, we have considered the case where the solutions are unbounded. Finally, we have supported our theoretical results.

Author Contribution Statements The authors contributed equally to the paper. All authors read and approved the final version of the paper.

Declaration of Competing Interests The authors declare that they have no competing interests.

\section{REFERENCES}

[1] Bede, B., Mathematics of Fuzzy Sets and Fuzzy Logic, Springer, New York, 2013.

[2] Chrysafis, K. A., Papadopoulos, B. K., Papaschinopoulos, G., On the fuzzy difference equations of finance, Fuzzy Sets Syst., 159 (2008), 3259-3270. https://doi:10.1016/j.fss.2008.06.007

[3] Deeba, E., De Korvin, A., Koh, E. L., A fuzzy difference equation with an application, J. Differ. Equ. Appl., 2 (1996), 365-374. https://doi.org/10.1080/10236199608808071

[4] Deeba, E., De Korvin, A., Analysis by fuzzy difference equations of a model of $\mathrm{CO}_{2}$ level in blood, Appl. Math. Lett., 12 (1999), 33-40. https://doi.org/10.1016/S0893-9659(98)00168-2

[5] El-Owaidy, H. M., Ahmed, A. M., Youssef, A. M., The dynamics of the recursive sequence $x_{n+1}=\left(\alpha x_{n-1}\right) /\left(\beta+\gamma x_{n-2}^{p}\right)$, Appl. Math. Lett., 18(9) (2005), 1013-1018. https://doi.org/10.1016/j.aml.2003.09.014

[6] Elsayed, E. M., On the solutions and periodic nature of some systems of difference equations, Int. J. Biomath., 7 (2014), 26 pages. https://doi.org/10.1142/S1793524514500673 
[7] Gümüş, M., Soykan, Y., Global character of a six-dimensional nonlinear system of difference equations, Discrete Dyn. Nat. Soc., Article ID 6842521 (2016), 7 pages. https://doi.org/10.1155/2016/6842521

[8] Klir, G., Yuan, B., Fuzzy Sets and Fuzzy Logic Theory and Applications, Prentice Hall, New Jersey, 1995.

[9] Hatir, E., Mansour, T., Yalcinkaya, I., On a fuzzy difference equation, Util. Math., 93 (2014), 135-151.

[10] Negoita, C. V., Ralescu, D., Applications of Fuzzy Sets to Systems Analysis, Birkhauser, Verlag, Besel, 1975.

[11] Papaschinopoulos, G., Papadopoulos, B. K., On the fuzzy difference equation $x_{n+1}=A+$ B/ $x_{n}$, Soft Comput., 6 (2002), 456-461. https://doi.org/10.1007/s00500-001-0161-7

[12] Papaschinopoulos, G., Papadopoulos, B. K., On the fuzzy difference equation $x_{n+1}=$ $A+x_{n} / x_{n-m}$, Fuzzy Sets Syst., 129 (2002), 73-81. https://doi.org/10.1016/S01650114(01)00198-1

[13] Stefanidou, G., Papaschinopoulos, G., A fuzzy difference equation of a rational form, $J$. Nonlinear Math. Phys., 12 (2005), 300-315. https://doi.org/10.2991/jnmp.2005.12.s2.21

[14] Pielou, E. C., Population and Community Ecology: Principles and Methods, CRC Press, London, 1974.

[15] Popov, E. P., Automatic Regulation and Control, (Russian) Nauka, Moscow, 1966.

[16] Wu, C., Zhang, B., Embedding problem of noncompact fuzzy number space $E^{\sim}$, Fuzzy Sets Syst., 105 (1999), 165-169. https://doi.org/10.1016/S0165-0114(97)00218-2

[17] Yalcinkaya, I., Atak, N., Tollu, D. T., On a third-order fuzzy difference equation, J. Prime Res. Math., 17(1) (2021), 59-69. http://jprm.sms.edu.pk/on-a-third-order-fuzzy-differenceequation/ 